Document downloaded from:

http://hdl.handle.net/10251/83249

This paper must be cited as:

Cantó Colomina, R.; Pelaez, MJ.; Urbano Salvador, AM. (2016). On the characterization of totally nonpositive matrices. SeMA Journal. 73(4):347-368. doi:10.1007/s40324-016-0073-1.

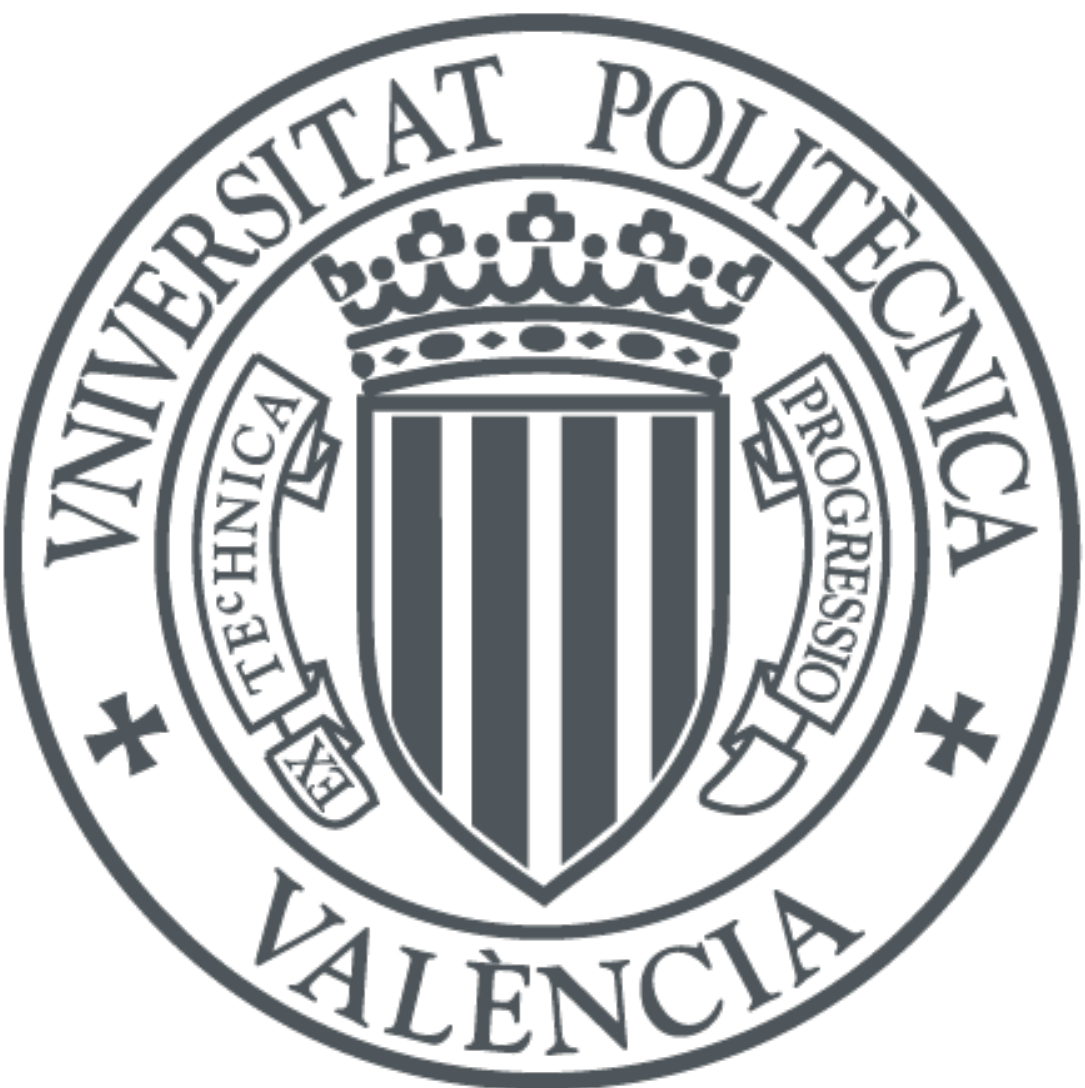

The final publication is available at

http://dx.doi.org/10.1007/s40324-016-0073-1

Copyright Springer

Additional Information

"The final publication is available at Springer via http://dx.doi.org/10.1007/s40324-016-00731 " 


\title{
On the characterization of totally nonpositive matrices
}

\section{Rafael Cantó · María J. Peláez •}

\author{
Ana M. Urbano
}

Received: date / Accepted: date

\begin{abstract}
A nonpositive real matrix $A=\left(a_{i j}\right)_{1 \leq i, j \leq n}$ is said to be totally nonpositive (negative) if all its minors are nonpositive (negative) and it is abbreviated as t.n.p. (t.n.). In this work a bidiagonal factorization of a nonsingular t.n.p. matrix $A$ is computed and it is stored in an matrix represented by $\mathcal{B D}_{(\text {t.n.p.) }}(A)$ when $a_{11}<0$ (or $\mathcal{B D}_{(\text {zero })}(A)$ when $\left.a_{11}=0\right)$. As a converse result, an efficient algorithm to know if an matrix $\mathcal{B D}$ (t.n.p.) $(A)\left(\mathcal{B D}_{(\text {zero })}(A)\right)$ is the bidiagonal factorization of a t.n.p. matrix with $a_{11}<0\left(a_{11}=0\right)$ is given. Similar results are obtained for t.n. matrices using the matrix $\mathcal{B D}_{(t . n .)}(A)$, and these characterizations are extended to rectangular t.n.p. (t.n.) matrices. Finally, the bidiagonal factorization of the inverse of a nonsingular t.n.p. (t.n.) matrix $A$ is directly obtained from $\mathcal{B D}_{(t . n . p .)}(A)\left(\mathcal{B D}_{(t . n .)}(A)\right)$.
\end{abstract}

Keywords Totally nonpositive matrix · totally negative matrix · inverse · bidiagonal factorization

Mathematics Subject Classification (2000) 15A15 $15 \mathrm{~A} 23 \cdot 65 \mathrm{~F} 40$

\section{Introduction}

A real matrix is called totally nonpositive (negative) if all its minors are nonpositive (negative) and it is abbreviated as t.n.p. (t.n.), see e.g. [4,5,7-9, 17, 19,

This research was supported by the Spanish DGI grant MTM2013-43678-P and by the Chilean program FONDECYT 1100029.

Rafael Cantó · Ana M. Urbano

Institut de Matemàtica Multidisciplinar, Universitat Politècnica de València, 46022 València, Spain. E-mail: rcanto@mat.upv.es, amurbano@mat.upv.es

María J. Peláez

Departamento de formación, ZLAB Innovación tecnológica S.L., Zaragoza, Spain.

E-mail: mpelaez-math@gmail.com 
25]. These matrices can be considered as a generalization of the partially negative matrices, that is, matrices with all its principal minors negative, which are called $N$-matrices in economic models [3,22]. If, instead, all minors of a matrix are nonnegative (positive) the matrix is called totally nonnegative (positive) and it is abbreviated as TP (STP). These matrices have a wide variety of applications and they have been studied by several authors $[1,6,10-14,16$, $23,24]$.

Recently, in [2] an algorithmic characterization of nonsingular almost strictly totally negative matrices is given through the Neville elimination. For t.n.p. matrices, Huang [18] obtained a bidiagonal factorization of a nonsingular t.n.p. matrix from the bidiagonal factorization of its inverse.

Now, in this paper we directly compute a bidiagonal factorization of a nonsingular t.n.p. matrix, different from the bidiagonal factorizations given in [18]. The main goal consists of directly compute the factorization using the entries of the t.n.p. matrix $A$ by applying the Neville elimination process without pivoting.

The paper is organized as follows: in Section 2, we propose a bidiagonal factorization of nonsingular t.n.p. matrices and we collect all elements of this bidiagonal factorization in the square matrix $\mathcal{B D}_{(\text {t.n.p.) }}(A)$ when $a_{11}<0$ (or $\mathcal{B D}_{(\text {zero })}(A)$ when $\left.a_{11}=0\right)$. Furthermore, we introduce two algorithms with quadratic cost that allow us to check if an matrix $\mathcal{B D}_{(\text {t.n.p. })}(A)\left(\mathcal{B D}_{(\text {zero })}(A)\right)$, with some specific properties, is the bidiagonal factorization of a t.n.p. matrix with $a_{11}<0\left(a_{11}=0\right)$. In Section 3, from the bidiagonal factorization of a nonsingular t.n.p. matrix $A$ we determine the bidiagonal factorization of the inverse of $A$. Finally, in Section 4 we extend the results of Section 2 to rectangular t.n.p. (t.n.) matrices with arbitrary rank.

\section{Bidiagonal factorization of nonsingular t.n.p. matrices}

Let $A=\left(a_{i j}\right) \in \mathbb{R}^{n \times n}$ be a nonsingular t.n.p. matrix. It is well-known, see [4], that the entries of $A$ are $a_{i j}<0$, except for $a_{11} \leq 0$ and $a_{n n} \leq 0$. In this section we obtain a bidiagonal factorization of $A$ applying Neville elimination and depending on $a_{11}<0$ or $a_{11}=0$. Neville elimination is a classical method alternative to Gaussian elimination and consists of making zeros in a column of a matrix by adding to each row an appropriate multiple of the precedent one, instead of using just a row with a fixed pivot as in Gaussian elimination (for a complete matricial description see [15]). This elimination technique plays an important role when dealing with sign regular matrices including totally nonnegative and totally nonpositive matrices and it has been used to get the factorization of a nonsingular TP matrix as a product of bidiagonal matrices (see $[12])$.

From now on, we denote by $E_{i}(x)$ a bidiagonal matrix which differs from the identity matrix only in its $(i, i-1)$ entry $x$ (see, for example, [15]). 
2.1 A t.n.p. matrix with a negative $(1,1)$-entry

A well-known result on nonsingular t.n.p. matrices with a negative $(1,1)$-entry proves that they have a unique $L D U$ factorization applying Neville elimination without pivoting [4]. Using this result, it is possible to construct a bidiagonal factorization of this class of matrices.

Theorem 1 Let $A=\left(a_{i j}\right) \in \mathbb{R}^{n \times n}$ be a nonsingular matrix with $a_{11}<0$ and $a_{n n} \leq 0$. Then, $A$ is a t.n.p. matrix if and only if $A$ admits the unique bidiagonal factorization

$$
A=F_{n-1} F_{n-2} \ldots F_{1} D G_{1} G_{2} \ldots G_{n-1}
$$

where $D=\operatorname{diag}\left(-d_{1}, d_{2}, \ldots, d_{n}\right)$, with $d_{1}, d_{2}, \ldots, d_{n}>0$, and for $i=1,2, \ldots$, $n-1, F_{i}\left(G_{i}\right)$ are the unit lower (upper) bidiagonal TP matrices defined by

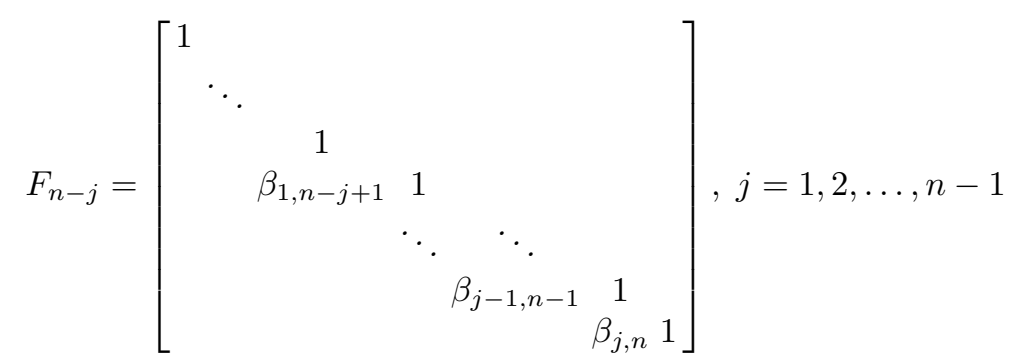

with $\beta_{1,2}>0, \ldots, \beta_{1, n}>0$ and if $\beta_{s, t}=0$ then $\beta_{s, h}=0$, for all $h>t$, and

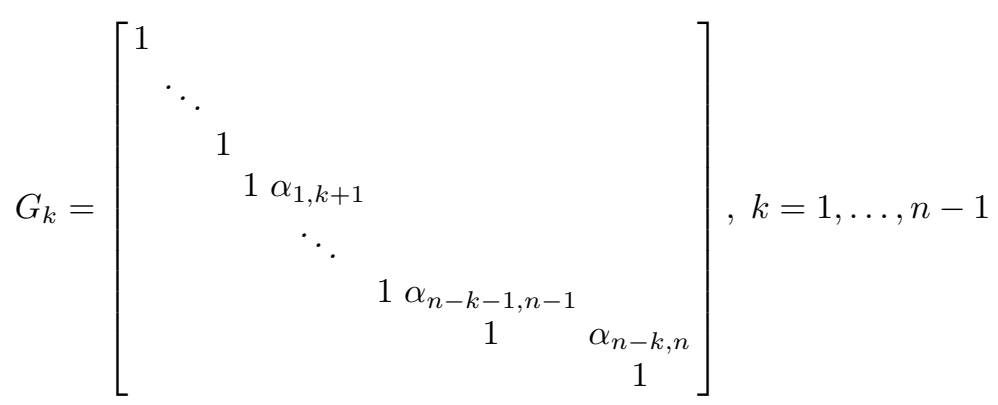

with $\alpha_{1,2}>0, \ldots, \alpha_{1, n}>0$, and if $\alpha_{s, t}=0$ then $\alpha_{s, h}=0$, for all $h>t$.

Proof By [4, Theorem 2.1], if $A$ is a t.n.p. matrix with $a_{11}<0$ admits the unique factorization $A=L D U$, where $L$ is a unit lower triangular TP matrix, $D=\operatorname{diag}\left(-d_{1}, d_{2}, \ldots, d_{n}\right)$, with $d_{i}>0$ for $i=1,2, \ldots, n$, and $U$ is a unit upper triangular TP matrix. Since $L$ is a TP matrix by [12, equation (3.1) pp. 117 ] we know that it admits the unique bidiagonal factorization,

$$
L=\left[E_{n}\left(\beta_{1, n}\right)\right]\left[E_{n-1}\left(\beta_{1, n-1}\right) E_{n}\left(\beta_{2, n}\right)\right] \ldots\left[E_{2}\left(\beta_{1,2}\right) E_{3}\left(\beta_{2,3}\right) \ldots E_{n}\left(\beta_{n-1, n}\right)\right]
$$


where $\beta_{1,2}>0, \ldots, \beta_{1, n}>0$ and if $\beta_{s, t}=0$ then $\beta_{s, h}=0, \forall h>t$. Multiplying the $\left(E_{i}\right)$ 's inside the brackets we obtain the bidiagonal matrices

$$
F_{n-j}=E_{n-j+1}\left(\beta_{1, n-j+1}\right) E_{n-j+2}\left(\beta_{2, n-j+2}\right) \ldots E_{n}\left(\beta_{j, n}\right)
$$

given by the expression and conditions (2).

Analogously, the TP matrix $U$ admits the unique bidiagonal factorization,

$U=\left[E_{n}^{T}\left(\alpha_{n-1, n}\right) \ldots E_{3}^{T}\left(\alpha_{2,3}\right) \ldots E_{2}^{T}\left(\alpha_{1,2}\right)\right] \ldots\left[E_{n}^{T}\left(\alpha_{2, n}\right) E_{n-1}^{T}\left(\alpha_{1, n-1}\right)\right]\left[E_{n}^{T}\left(\alpha_{1, n}\right)\right]$,

where $\alpha_{1,2}>0, \ldots, \alpha_{1, n}>0$ and if $\alpha_{s, t}=0$ then $\alpha_{s, h}=0, \forall h>t$. Multiplying the $\left(E_{i}^{T}\right)$ 's inside the brackets we obtain the bidiagonal matrices

$$
G_{k}=E_{n}^{T}\left(\alpha_{n-k, n}\right) \ldots E_{k+2}^{T}\left(\alpha_{2, k+2}\right) E_{k+1}^{T}\left(\alpha_{1, k+1}\right)
$$

given by the expression and conditions (3). Therefore, $A$ admits the unique bidiagonal factorization given in (1).

Conversely, from the bidiagonal factorization

$$
A=F_{n-1} F_{n-2} \ldots F_{1} D G_{1} G_{2} \ldots G_{n-1}
$$

we construct $L$ and $U$ in the following form

$$
\begin{aligned}
L & =F_{n-1} F_{n-2} \ldots F_{2} F_{1} \\
U & =G_{1} G_{2} \ldots G_{n-2} G_{n-1}
\end{aligned}
$$

Since $F_{i}\left(G_{i}\right), i=1,2, \ldots, n-1$, are unit lower (upper) bidiagonal TP matrices, then $L$ and $U$ are unit lower (upper) triangular TP matrices. Then, we have that $A=L D U$ is a t.n.p. matrix by [4, Theorem 2.5].

By [4] and with an analogous reasoning, a similar factorization is obtained for t.n. matrices with all parameters $\alpha_{i, j}$ and $\beta_{i, j}$ positive.

Corollary 1 Let $A=\left(a_{i j}\right) \in \mathbb{R}^{n \times n}$ be a nonsingular matrix with $a_{n n}<0$. A is a t.n. matrix if and only if $A$ admits the unique bidiagonal factorization

$$
A=F_{n-1} F_{n-2} \ldots F_{1} D G_{1} G_{2} \ldots G_{n-1}
$$

where $D=\operatorname{diag}\left(-d_{1}, d_{2}, \ldots, d_{n}\right)$, with $d_{i}>0, F_{i}\left(G_{i}\right)$, for $i=1,2, \ldots, n$, are unit lower (upper) bidiagonal TP matrices given by expressions (2) and (3), respectively, and satisfying the conditions $\beta_{i, j}>0$ and $\alpha_{i, j}>0$, for all $i=1,2, \ldots, n-1$ and $j=2,3, \ldots, n$.

Koev [20,21] denotes the bidiagonal decomposition of a TP matrix $A$ as $B D(A)$ and he stores the nontrivial entries of $B D(A)$ compactly in an $n \times n$ matrix which he also refers to as $B D(A)$. Now, we extend this notation to t.n.p. matrices. 
Definition 1 Let $A$ be an $n \times n$ nonsingular t.n.p. matrix with $a_{11}<0$ and $a_{n n} \leq 0$. Using Theorem 1 , we denote by $\mathcal{B D}_{(t . n . p .)}(A)$ the obtained bidiagonal decomposition of $A$ and we store the entries of $\mathcal{B D}_{(t . n . p .)}(A)$ in the $n \times n$ matrix

$$
\mathcal{B D}_{(\text {t.n.p. })}(A)=\left[\begin{array}{cccccc}
-d_{1} & \alpha_{1,2} & \alpha_{1,3} & \cdots & \alpha_{1, n-1} & \alpha_{1, n} \\
\beta_{1,2} & d_{2} & \alpha_{2,3} & \cdots & \alpha_{2, n-1} & \alpha_{2, n} \\
\beta_{1,3} & \beta_{2,3} & d_{3} & \cdots & \alpha_{3, n-1} & \alpha_{3, n} \\
\vdots & \vdots & \vdots & & \vdots & \vdots \\
\beta_{1, n-1} & \beta_{2, n-1} & \beta_{3, n-1} & \cdots & d_{n-1} & \alpha_{n-1, n} \\
\beta_{1, n} & \beta_{2, n} & \beta_{3, n} & \cdots & \beta_{n-1, n} & d_{n}
\end{array}\right]
$$

where

$$
\begin{aligned}
d_{i} & >0, \quad \beta_{i, j} \geq 0, \quad \alpha_{i, j} \geq 0 \quad \text { for } \quad i, j=1,2, \ldots, n, \\
\beta_{1, j} & >0, \quad \alpha_{1, j}>0 \quad \text { for } j=2,3, \ldots, n, \\
\text { if } \quad \beta_{i, j} & =0, \quad\left(\alpha_{i, j}=0\right) \Longrightarrow \beta_{i, h}=0, \quad\left(\alpha_{i, h}=0\right) \text { for all } h>j .
\end{aligned}
$$

If $A$ is a t.n. matrix then we obtain the bidiagonal decomposition $\mathcal{B D}_{(t . n .)}(A)$, with $\beta_{i, j}>0$ and $\alpha_{i, j}>0$ for all $i=1,2, \ldots, n-1$ and $j=2,3, \ldots, n$.

The converse of this result is not true in general, that is, an $n \times n$ matrix $\mathcal{B D}_{(\text {t.n.p.) }}(A)$, with the conditions given by $(5),(6)$ and $(7)$, is not always the bidiagonal factorization of a t.n.p. matrix $A$ with $a_{11}=-d_{1}<0$, see the following example. This is an important difference between TP (STP) matrices and t.n.p. (t.n.) matrices.

Example 1 Consider the matrix

$$
\mathcal{B D}_{(t . n . p .)}(A)=\left[\begin{array}{rrrrr}
-3 & 2 & 2 & 1 & 2 \\
4 & 1 & 3 & 1 & 1 \\
5 & 2 & 2 & 1 & 0 \\
1 & 3 & 1 & 1 & 1 \\
2 & 3 & 0 & 2 & 1
\end{array}\right]
$$

Note that its entries verify the conditions (5), (6) and (7). Following Theorem 1 , we construct the bidiagonal matrices $F_{i}$ and $G_{j}$, for $i, j=1,2,3,4$, and the diagonal matrix $D$. Then, we have that

$$
A=\underbrace{F_{4} F_{3} F_{2} F_{1}}_{L} D \underbrace{G_{1} G_{2} G_{3} G_{4}}_{U}=\left[\begin{array}{rrrrr}
-3 & -6 & -12 & -12 & -24 \\
-12 & -23 & -43 & -40 & -77 \\
-60 & -113 & -203 & -178 & -331 \\
-60 & -107 & -165 & -105 & -149 \\
-120 & -196 & -216 & 11 & 257
\end{array}\right],
$$

which is not a t.n.p. matrix because the $(5,4)$ and $(5,5)$ entries are positive. 
To know if an $n \times n$ matrix $\mathcal{B D}_{(\text {t.n.p. })}(A)$, with the conditions given by (5), (6) and (7), is the bidiagonal factorization of a t.n.p. matrix $A$ with $a_{11}<0$ we only need to compute $a_{n n}$, see [4, Theorem 2.4]. If $a_{n n} \leq 0$ then it is and if $a_{n n}>0$ it is not. Therefore, we present an algorithm that computes $a_{n n}$ directly from the elements of $\mathcal{B D}_{(\text {t.n.p. })}(A)$ without obtaining matrices $F_{i}$ and $G_{j}, i, j=1,2, \ldots, n-1$. From $\mathcal{B D}_{(t . n . p .)}(A)$ we compute $l^{(n)}$ and $u_{(n)}$, that is, the last row of $L$, and the last column of $U$, respectively, and after we obtain $a_{n n}$ as the product $l^{(n)} D u_{(n)}$. The following equalities to construct the last row of $L, l^{(n)}=\left[l_{n, 1}, l_{n, 2}, \ldots, l_{n, n-1}, 1\right]$ from $\mathcal{B D}_{(t . n . p .)}(A)$ are used in Algorithm 1:

$$
\begin{aligned}
l_{n, n-1}= & \underbrace{\beta_{1, n}}_{h_{1}^{(n-1)}}+\underbrace{\beta_{2, n}}_{h_{2}^{(n-1)}}+\cdots+\underbrace{\beta_{n-2, n}}_{h_{n-2}^{(n-1)}}+\underbrace{\beta_{n-1, n}}_{h_{n-1}^{(n-1)}} \\
l_{n, n-2}= & \underbrace{\beta_{1, n-1} h_{1}^{(n-1)}}_{h_{1}^{(n-2)}}+\underbrace{\beta_{2, n-1}\left(h_{1}^{(n-1)}+h_{2}^{(n-1)}\right)}_{h_{2}^{(n-2)}}+\cdots+ \\
& +\cdots+\underbrace{\beta_{n-2, n-1}\left(h_{1}^{(n-1)}+h_{2}^{(n-1)}+\cdots+h_{n-3}^{(n-1)}+h_{n-2}^{(n-1)}\right)}_{h_{n-2}^{(n-2)}} \\
l_{n, n-3}= & \underbrace{\beta_{1, n-2} h_{1}^{(n-2)}}_{h_{1}^{(n-3)}}+\underbrace{\beta_{2, n-2}\left(h_{1}^{(n-2)}+h_{2}^{(n-2)}\right)}_{h_{2}^{(n-3)}}+\cdots+ \\
& +\cdots+\underbrace{\beta_{n-3, n-2}\left(h_{1}^{(n-2)}+h_{2}^{(n-2)}+\cdots+h_{n-4}^{(n-2)}+h_{n-3}^{(n-2)}\right)}_{h_{n-3}^{(n-3)}} \\
l_{n, 2}= & \underbrace{\beta_{1,3} h_{1}^{(3)}}_{h_{1}^{(2)}}+\underbrace{\beta_{2,3}\left(h_{1}^{(3)}+h_{2}^{(3)}\right)}_{h_{2}^{(2)}} \\
l_{n, 1}= & \underbrace{\beta_{1}^{(2)}}_{1,2} h_{1}^{(2)}
\end{aligned}
$$


$\overline{\text { Algorithm } 1 \text { (LastRow) It computes the last row of } L \text { directly from the }}$ elements of an $n \times n$ matrix $\mathcal{B D}_{(\text {t.n.p.) }}(A)$ in form (4) with the properties given by (5), (6) and (7).

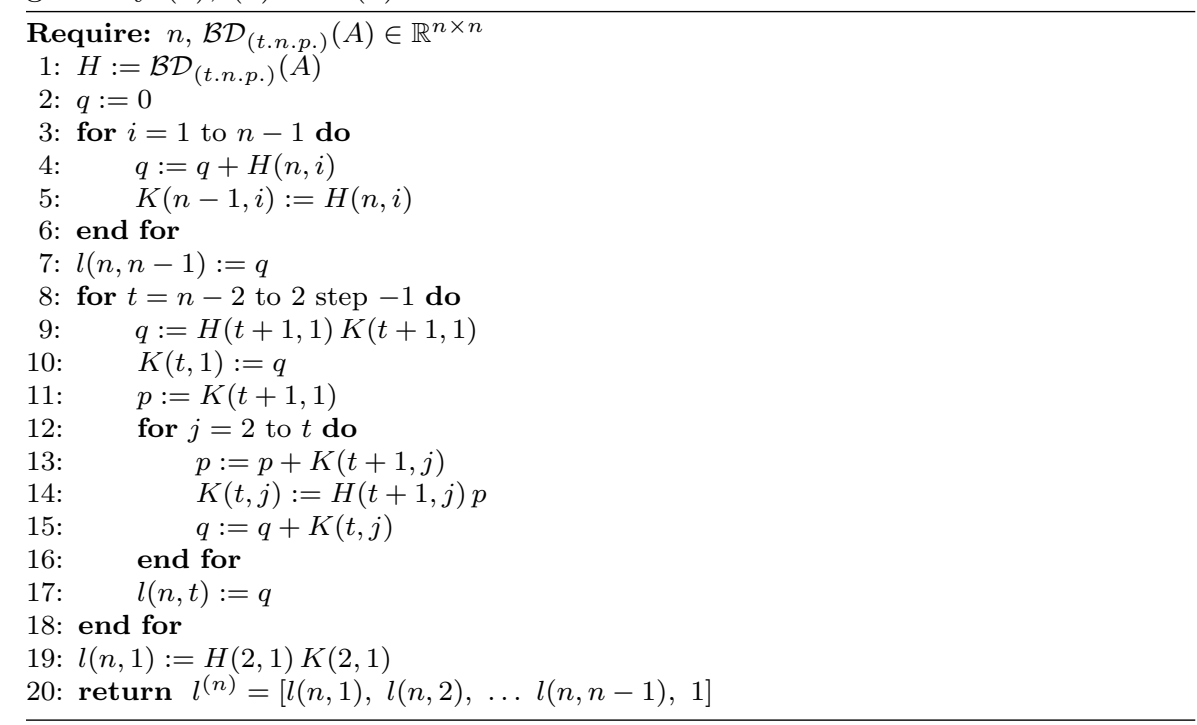

Theorem 2 The computational cost of Algorithm 1 is $\frac{3}{2} n^{2}-\frac{11}{2} n+5$.

Proof Analyzing Algorithm 1 we observe the following operations:

- for of line 3 runs $n-1$ times with one sum each time.

- for of line 8 runs $n-3$ times. In each time there is one arithmetic operation and an inner for in line 12, which runs $t-1$ times with 3 arithmetic operations each time.

- Finally, a product in line 19.

Then, the total computational cost to obtain $l^{(n)}$ is

$$
O=n-1+\sum_{t=2}^{n-2}\left(1+\sum_{j=2}^{t} 3\right)+1=\frac{3}{2} n^{2}-\frac{11}{2} n+6
$$

Example 2 Consider the following $5 \times 5$ matrix

$$
\mathcal{B D}_{(\text {t.n.p. })}(A)=\left[\begin{array}{rrrrr}
-4 & 2 & 2 & 1 & 2 \\
4 & 1 & 3 & 1 & 1 \\
5 & 2 & 2 & 1 & 0 \\
1 & 3 & 1 & 1 & 1 \\
2 & 3 & 0 & 2 & 1
\end{array}\right]
$$

by applying Algorithm 1 we obtain 


$$
\begin{aligned}
l(5,4) & =\beta_{1,5}+\beta_{2,5}+\beta_{3,5}+\beta_{4,5}=2+3+0+2=7 \\
l(5,3) & =\beta_{1,4} \beta_{1,5}+\beta_{2,4}\left(\beta_{1,5}+\beta_{2,5}\right)+\beta_{3,4}\left(\beta_{1,5}+\beta_{2,5}+\beta_{3,5}\right) \\
& =\beta_{1,4} \beta_{1,5}+\beta_{2,4} \beta_{1,5}+\beta_{2,4} \beta_{2,5}+\beta_{3,4} \beta_{1,5}+\beta_{3,4} \beta_{2,5}+\beta_{3,4} \beta_{3,5}=22,
\end{aligned}
$$

graphically is,

\begin{tabular}{llll|llllll}
$-d_{1}$ & & & & -4 & & & & \\
$\beta_{12}$ & $d_{2}$ & & & & 4 & 1 & & & \\
$\beta_{13}$ & $\beta_{23}$ & $d_{3}$ & & & 5 & 2 & 2 & & \\
$\beta_{14}$ & $\beta_{24}$ & $\beta_{34}$ & $d_{4}$ & & 1 & 3 & 1 & 1 & \\
$\downarrow$ & $\downarrow$ & $\downarrow$ & & & $\downarrow$ & $\downarrow$ & $\downarrow$ & & \\
$\beta_{15}$ & $\beta_{25}$ & $\beta_{35}$ & $\beta_{45}$ & $d_{5}$ & 2 & 3 & 0 & 2 & 1
\end{tabular}

$$
\begin{aligned}
l_{53} & =\beta_{14} \beta_{15}+\beta_{24} \beta_{15}+\beta_{24} \beta_{25}+\beta_{34} \beta_{15}+\beta_{34} \beta_{25} \\
& +\beta_{34} \beta_{35}=1.2+3.2+3.3+1.2+1.3=22
\end{aligned}
$$

\begin{tabular}{|c|c|c|c|}
\hline$-d_{1}$ & -4 & & \\
\hline$\beta_{12} \quad d_{2}$ & 41 & & \\
\hline $\begin{array}{lll}\beta_{13} & \beta_{23} & d_{3} \\
\downarrow & \downarrow & \end{array}$ & $\begin{array}{lr}5 & 2 \\
\downarrow & \downarrow\end{array}$ & 2 & \\
\hline $\begin{array}{llll}\beta_{14} & \beta_{24} & \beta_{34} & d_{4}\end{array}$ & 13 & 1 & 1 \\
\hline $\begin{array}{lllll}\beta_{15} & \beta_{25} & \beta_{35} & \beta_{45} & d_{5}\end{array}$ & 23 & 0 & 2 \\
\hline
\end{tabular}

$$
\begin{aligned}
l(5,2) & =\beta_{1,3} \beta_{1,4} \beta_{1,5}+\beta_{2,3}\left(\beta_{1,4} \beta_{1,5}+\beta_{2,4}\left(\beta_{1,5}+\beta_{2,5}\right)\right) \\
& =\beta_{1,3} \beta_{1,4} \beta_{1,5}+\beta_{2,3} \beta_{1,4} \beta_{1,5}+\beta_{2,3} \beta_{2,4} \beta_{1,5}+\beta_{2,3} \beta_{2,4} \beta_{2,5}=44,
\end{aligned}
$$

and graphically is, 
Finally,

$$
l(5,1)=\beta_{1,2} \beta_{1,3} \beta_{1,4} \beta_{1,5}=40 .
$$

and graphically,

$$
\begin{aligned}
& \begin{array}{cccc|cccccc}
-d_{1} & & & & & -4 & & & & \\
\beta_{12} & d_{2} & & & & & & & & \\
\downarrow & & & & & & & & \\
\beta_{13} & \beta_{23} & d_{3} & & & & & & \\
\downarrow & & & 2 & 2 & & \\
\beta_{14} & \beta_{24} & \beta_{34} & d_{4} & & 1 & 3 & 1 & 1 & \\
\downarrow & & & & & \downarrow & & & & \\
\beta_{15} & \beta_{25} & \beta_{35} & \beta_{45} & d_{5} & 2 & 3 & 0 & 2 & 1
\end{array} \\
& l_{51}=\beta_{12} \beta_{13} \beta_{14} \beta_{15}=4.5 .1 .2=40
\end{aligned}
$$

Then, $l^{(5)}=[l(5,1), l(5,2), l(5,3), l(5,2), 1]=[40,44,22,7,1]$.

Now, the following algorithm checks if an square matrix $\mathcal{B D}_{(\text {t.n.p. }}(A)$ in the form (4), with the conditions given by equations (5), (6) and (7), is the bidiagonal factorization of a t.n.p. matrix $A$ with $a_{11}=-d_{1}<0$. Its computational costs is $\mathbb{O}\left(n^{2}\right)$.

Algorithm $2\left(\right.$ IsTnp) It tests if an square matrix $\mathcal{B} \mathcal{D}_{(t . n . p .)}(A)$ in form $(4)$ with the properties given by equations $(5),(6)$ and $(7)$, is the bidiagonal factorization of a t.n.p. matrix $A$ with $a_{11}=-d_{1}<0$

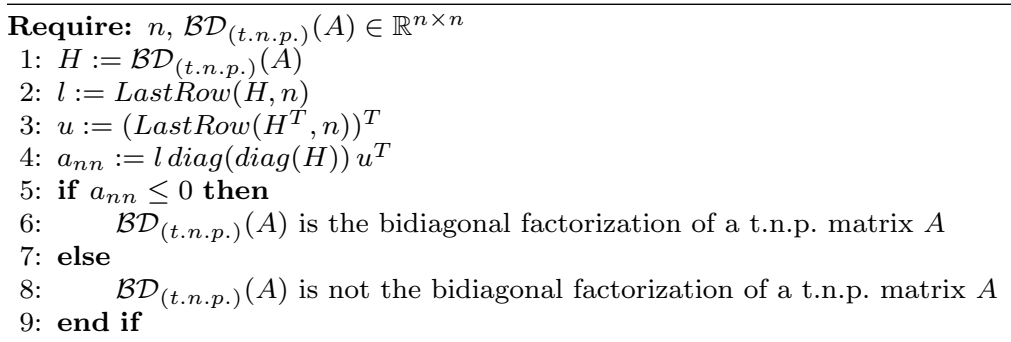

Corollary 2 The computational cost of Algorithm 2 is $3 n^{2}-8 n+7$.

Proof To compute the element $a_{n n}$ as follows,

$$
\begin{aligned}
a_{n n} & =l^{(n)} D u_{(n)}=-d_{1} l_{n, 1} u_{1, n}+\underbrace{\left(d_{2} l_{n, 2} u_{2, n}+\cdots+d_{n-1} l_{n, n-1} u_{n-1, n}+d_{n}\right)}_{x} \\
& =-d_{1} l_{n, 1} u_{1, n}+x
\end{aligned}
$$


it is necessary $3(n-1)$ operations between products and sums. Then, the computational cost is

$$
\begin{aligned}
O & =\underbrace{\left(\frac{3}{2} n^{2}-\frac{11}{2} n+5\right)}_{\text {to compute } l}+\underbrace{\left(\frac{3}{2} n^{2}-\frac{11}{2} n+5\right)}_{\text {to compute } u}+3(n-1) \\
& =3 n^{2}-8 n+7 .
\end{aligned}
$$

Remark 1 Note that if an square matrix $\mathcal{B D}_{(t . n .)}(A)$ in form (4) has all its elements greater than zero except for the negative $(1,1)$-entry, Algorithms 1 and 2 also allow us to check if this matrix is the bidiagonal factorization of a t.n. matrix $A$.

Remark 2 Algorithms 1 and 2 check in a very efficient way if $\mathcal{B D}_{(\text {t.n.p.) }}(A)$ $\left(\mathcal{B D}_{(t . n .)}(A)\right)$ is the bidiagonal factorization of a t.n.p. (t.n.) matrix $A$ with a negative $(1,1)$-entry. Therefore, they are useful to generate this kind of matrices.

Example 3 In Example 2, we have obtained

$$
l^{(5)}=[l(5,1), l(5,2), l(5,3), l(5,2), 1]=[40,44,22,7,1] .
$$

Applying the same procedure to $\left(\mathcal{B D}_{(\text {t.n.p. })}(A)\right)^{T}$ we obtain $u_{(5)}=[8,19,8,4,1]^{T}$. Since,

$$
D=\operatorname{diag}\left(\mathcal{B D}_{(t . n . p .)}(A)=\operatorname{diag}(-4,1,2,1,1)\right.
$$

then, $a_{5,5}=l^{(5)} D u_{(5)}=-63<0$, that is, $\mathcal{B D}_{(t . n . p .)}(A)$ is the bidiagonal factorization of a t.n.p. matrix.

Note that, the only difference between the matrices of Examples 1 and 2 is the $(1,1)$-entry. If $a_{11}=-3$ then the matrix $\mathcal{B D}_{(t . n . p .)}(A)$ is not the bidiagonal factorization of a t.n.p. matrix. Otherwise, if $a_{11}=-4$ then the answer is affirmative.

2.2 A t.n.p. matrix with a zero $(1,1)$-entry

Now, we consider nonsingular t.n.p. matrices with $a_{11}=0$. For these matrices, a distinct bidiagonal factorization can be obtained by using the matrix $Q$ in the constructive process, where $Q$ is the identity matrix except for the first $2 \times 2$ principal block equal to $\left(\begin{array}{rr}0 & -1 \\ -1 & 0\end{array}\right)$. 
Theorem 3 Let $A=\left(a_{i j}\right) \in \mathbb{R}^{n \times n}$ be a nonsingular t.n.p. matrix with $a_{11}=0$. Then, $A$ can be uniquely factorized as

$$
A=E_{n}\left(\beta_{1, n}\right) E_{n-1}\left(\beta_{1, n-1}\right) \ldots E_{3}\left(\beta_{1,3}\right) Q F_{n-2} \ldots F_{1} D G_{1} G_{2} \ldots G_{n-1}
$$

where $D=\operatorname{diag}\left(d_{1}, d_{2}, \ldots, d_{n}\right)$, with $d_{i}>0$ for $i=1,2, \ldots, n$. $E_{t}$ is a bidiagonal matrix which differs from the identity only in its $(t, t-1)$ entry $\beta_{1 t}>0$, for $t=3,4, \ldots, n . F_{n-j}$ is the following nonnegative bidiagonal matrix

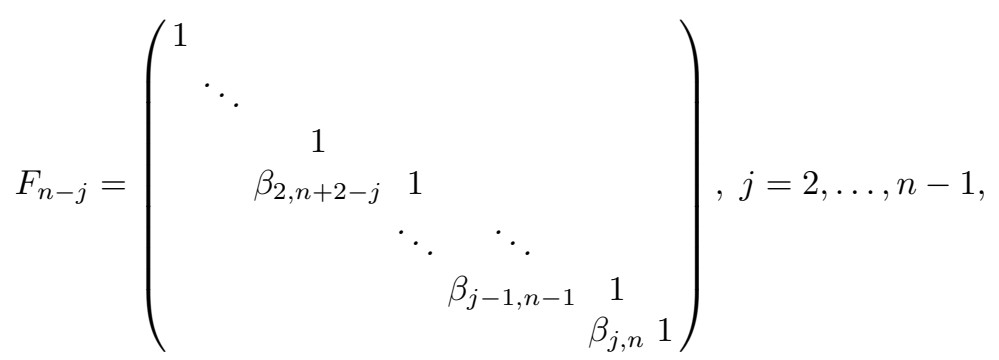

such that $\beta_{s, t}=0 \Rightarrow \beta_{s, h}=0 \forall h>t$, and $G_{k}$ is the following nonnegative bidiagonal matrix

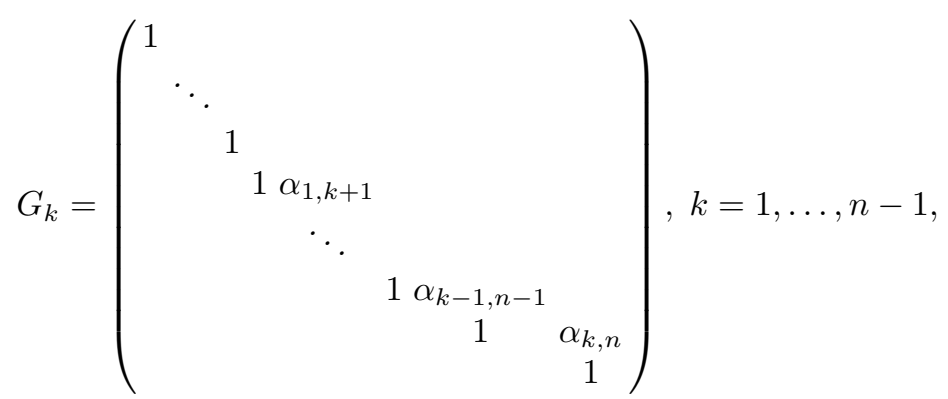

with $\alpha_{1,2}>0, \ldots, \alpha_{1, n}>0$ and such that $\alpha_{s, t}=0 \Rightarrow \alpha_{s, h}=0 \forall h>t$.

Proof Note that all entries $a_{i 1}<0$, for all $i=2,3, \ldots, n$ (see [7]). We apply the first iteration of the Neville elimination and multiply by the matrix $Q$. Then, we obtain the following matrix $A_{1}$,

$$
A_{1}=Q \underbrace{E_{3}\left(-\beta_{1,3}\right) \ldots E_{n-1}\left(-\beta_{1, n-1}\right) E_{n}\left(-\beta_{1, n}\right)}_{E^{(1)}} A=Q E^{(1)} A,
$$

or equivalently,

$$
A_{1}=\left(\begin{array}{ccccc}
-a_{2,1} & -a_{2,2} & \cdots & -a_{2, n-1} & -a_{2, n} \\
0 & -a_{1,2} & \cdots & -a_{1, n-1} & -a_{1, n} \\
0 & a_{3,2}^{(1)} & \cdots & a_{3, n-1}^{(1)} & a_{3, n}^{(1)} \\
\vdots & \vdots & & \vdots & \vdots \\
0 & a_{n-1,2}^{(1)} & \cdots & a_{n-1, n-1}^{(1)} & a_{n-1, n}^{(1)} \\
0 & a_{n, 2}^{(1)} & \cdots & a_{n, n-1}^{(1)} & a_{n, n}^{(1)}
\end{array}\right),
$$


with $a_{i, j}^{(1)} \geq 0$. The matrix $A_{1}$ is TP because if we apply Binet-Cauchy (see [13, Theorem 3.1]), then:

1. For all $k=1,2, \ldots, n$,

$$
\begin{aligned}
& \operatorname{det}\left(A_{1}\right)[1,2, \ldots, k]=\operatorname{det}\left(Q E^{(1)} A\right)[1,2, \ldots, k] \\
& =\sum_{\forall \gamma \in \mathcal{Q}_{k, n}} \operatorname{det}(Q)[1,2, \ldots, k \mid \gamma] \operatorname{det}\left(E^{(1)} A\right)[\gamma \mid 1,2, \ldots, k] \\
& =\operatorname{det}(Q)[1,2, \ldots, k] \operatorname{det}\left(E^{(1)} A\right)[1,2, \ldots, k] \\
& =-\operatorname{det}\left(E^{(1)} A\right)[1,2, \ldots, k] \\
& =-\sum_{\forall \gamma \in \mathcal{Q}_{k, n}} \operatorname{det}\left(E^{(1)}\right)[1,2, \ldots, k \mid \gamma] \operatorname{det}(A)[\gamma \mid 1,2, \ldots, k] \\
& =-\operatorname{det}\left(E^{(1)}\right)[1,2, \ldots, k] \operatorname{det}(A)[1,2, \ldots, k] \\
& =-\operatorname{det}(A)[1,2, \ldots, k]>0 .
\end{aligned}
$$

2. For all $\beta \in \mathcal{Q}_{k, n}, k=1,2, \ldots, n$,

$$
\begin{aligned}
& \operatorname{det}\left(A_{1}\right)[1,2, \ldots, k \mid \beta]=\operatorname{det}\left(Q E^{(1)} A\right)[1,2, \ldots, k \mid \beta] \\
& =\sum_{\forall \gamma \in \mathcal{Q}_{k, n}} \operatorname{det}(Q)[1,2, \ldots, k \mid \gamma] \operatorname{det}\left(E^{(1)} A\right)[\gamma \mid \beta] \\
& =\operatorname{det}(Q)[1,2, \ldots, k] \operatorname{det}\left(E^{(1)} A\right)[1,2, \ldots, k \mid \beta] \\
& =-\operatorname{det}\left(E^{(1)} A\right)[1,2, \ldots, k \mid \beta] \\
& =-\sum_{\forall \gamma \in \mathcal{Q}_{k, n}} \operatorname{det}\left(E^{(1)}\right)[1,2, \ldots, k \mid \gamma] \operatorname{det}(A)[\gamma \mid \beta] \\
& =-\operatorname{det}\left(E^{(1)}\right)[1,2, \ldots, k] \operatorname{det}(A)[1,2, \ldots, k \mid \beta] \\
& =-\operatorname{det}(A)[1,2, \ldots, k \mid \beta] \geq 0 .
\end{aligned}
$$

3. For all $\alpha \in \mathcal{Q}_{k, n}, k=1,2, \ldots, n$,

$$
\begin{aligned}
& \operatorname{det}\left(A_{1}\right)[\alpha \mid 1,2, \ldots, k]=\operatorname{det}\left(Q E^{(1)} A\right)[\alpha \mid 1,2, \ldots, k] \\
& =\operatorname{det}\left(Q E^{(1)} A\right)\left[\alpha_{1}, \alpha_{2}, \ldots, \alpha_{n} \mid 1,2, \ldots, k\right] \\
& =\left\{\begin{array}{l}
\alpha_{1}>1 \rightarrow=0 \\
\alpha_{1}=1 \rightarrow=\operatorname{det}\left(Q E^{(1)} A\right)\left[1, \alpha_{2}, \ldots, \alpha_{n} \mid 1,2, \ldots, k\right] \\
=-a_{21} \operatorname{det}\left(Q E^{(1)} A\right)\left[\alpha_{2}, \ldots, \alpha_{n} \mid 2, \ldots, k\right]
\end{array}\right.
\end{aligned}
$$

Using [5], if $\alpha_{2}>2$ we have that,

$$
\begin{gathered}
\operatorname{det}\left(Q E^{(1)} A\right)\left[\alpha_{2}, \ldots, \alpha_{n} \mid 2, \ldots, k\right] \geq 0 \\
\Downarrow \\
-a_{21} \operatorname{det}\left(Q E^{(1)} A\right)\left[\alpha_{2}, \ldots, \alpha_{n} \mid 2, \ldots, k\right] \geq 0
\end{gathered}
$$

Using [7], if $\alpha_{2}=2$ we have that,

$$
\begin{gathered}
\operatorname{det}\left(Q E^{(1)} A\right)\left[2, \ldots, \alpha_{n} \mid 2, \ldots, k\right] \geq 0 \\
\Downarrow \\
-a_{21} \operatorname{det}\left(Q E^{(1)} A\right)\left[2, \ldots, \alpha_{n} \mid 2, \ldots, k\right] \geq 0
\end{gathered}
$$


Therefore, $A_{1}$ is a TP matrix. Applying [12], $A_{1}$ can be decomposed uniquely as follows,

$$
A_{1}=F_{n-1} F_{n-2} \cdots F_{1} D G_{1} G_{2} \cdots G_{n-1}
$$

where $D=\operatorname{diag}\left(d_{1}, d_{2}, \ldots, d_{n}\right)$, with $d_{i}>0$ for $i=1,2, \ldots, n, F_{n-1}=I_{n}$. $F_{k}, G_{h}, k=1,2, \ldots, n-2, h=1,2, \ldots, n-1$ are analogous to matrices $(9)$ and (10), respectively. Then, $A$ can be decomposed uniquely as

$$
A=E_{n}\left(\beta_{1, n}\right) E_{n-1}\left(\beta_{1, n-1}\right) \cdots E_{3}\left(\beta_{1,3}\right) Q F_{n-2} \cdots F_{1} D G_{1} G_{2} \cdots G_{n-1} .
$$

If $A$ is a nonsingular t.n.p. matrix with $a_{11}=0$, then it is not possible to obtain a $L D U$ factorization by applying Neville elimination without pivoting. From the factorization (8) we can construct a quasi $L D U$ factorization of $A$, that is $A=L Q \tilde{L} D U$ where $L, \tilde{L}$ and $U$ are TP matrices, as in the case when $a_{11}<0$. This factorization and Theorem 4 allow us to give other characterization of this class of matrices similar to the expression obtained when $a_{11}<0$ (see [4]).

Corollary 3 Let $A \in \mathbb{R}^{n \times n}$ be a nonsingular t.n.p. matrix with $a_{11}=0$. Then, $A$ can be uniquely factorized as

$$
A=L Q \tilde{L} D U
$$

where $L$ and $\tilde{L}$ are unit lower triangular TP matrices, $D$ is a positive diagonal matrix and $U$ is a unit upper triangular TP matrix.

Proof Consider the factorization (8) of $A$. We define $L=E_{n}\left(\beta_{1, n}\right) E_{n-1}\left(\beta_{1, n-1}\right)$ $\ldots E_{3}\left(\beta_{1,3}\right), \tilde{L}=F_{n-2} \ldots F_{1}$ and $U=G_{1} G_{2} \ldots G_{n-1}$, then the result follows.

The converse of Theorem 3 is not true in general, because the $(n, n)$-entry of $A$ can be positive. Next result proves the converse by adding the necessary condition that the $(n, n)$-entry of $A$ must be nonpositive.

Theorem 4 Let $A=L Q \tilde{L} D U \in \mathbb{R}^{n \times n}$ be a matrix where $a_{n n} \leq 0$, L, $\tilde{L}(U)$ are unit lower (upper) triangular TP matrices with positive entries below (above) the main diagonal and $D=\operatorname{diag}\left(d_{1}, \ldots, d_{n}\right)$ with $d_{i}>0$, $i=1,2, \ldots, n$. Then, $A$ is t.n.p. with $a_{11}=0$.

Proof Using the matrix $H=\operatorname{diag}(-1,-1,1 \ldots, 1)$ we have

$$
\begin{aligned}
& L Q \tilde{L} D=L(Q \tilde{L} H)(H D) \\
& =L\left[\begin{array}{cccccc}
0 & 1 & & & \\
1 & 0 & & & \\
0 & -\tilde{l}_{3,2} & 1 & & \\
0 & -\tilde{l}_{4,2} & \tilde{l}_{4,3} & & \\
\vdots & \vdots & \vdots & & \\
0 & -\tilde{l}_{n-1,2} & \tilde{l}_{n-1,3} & \ldots & 1 \\
0 & -\tilde{l}_{n, 2} & \tilde{l}_{n, 3} & \ldots & \tilde{l}_{n, n-1} \\
0
\end{array}\right]\left[\begin{array}{ccccc}
-d_{1} & 0 & \ldots & 0 & 0 \\
0 & -d_{2} & \ldots & 0 & 0 \\
\vdots & \vdots & & \vdots & \vdots \\
0 & 0 & \ldots & d_{n-1} & 0 \\
0 & 0 & \ldots & 0 & d_{n}
\end{array}\right] \\
& =T \bar{D} .
\end{aligned}
$$

The matrix $T=L Q \tilde{L} H$ satisfies, 
1. The principal submatrix $T[3,4, \ldots, n]$ is TP.

2. The first elements of the first column $t_{i, 1}$, for $i=2,3, \ldots, n$, is positive.

3 . The elements of the second column $t_{i, 2}$, for $i=3,4, \ldots, n$ are nonpositive because, $\operatorname{det}(T[i \mid 2])=\operatorname{det}((L Q \tilde{L} H)[(i \mid 2])=-\operatorname{det}(L Q \tilde{L})([(i \mid 2]) \leq 0$.

4. For all $\alpha \in \mathcal{Q}_{k, n}, k=2,3, \ldots, n$,

$$
\begin{aligned}
\operatorname{det}(T[\alpha \mid 1,2, \ldots, k]) & =\operatorname{det}((L Q \tilde{L} H)[\alpha \mid 1,2, \ldots, k]) \\
& =\operatorname{det}((L Q \tilde{L})[\alpha \mid 1,2, \ldots, k]) \\
& =\sum_{\forall \gamma \in \mathcal{Q}_{k, n}} \operatorname{det}(L[\alpha \mid \gamma]) \operatorname{det}((Q \tilde{L})[\gamma \mid 1,2, \ldots, k]) .
\end{aligned}
$$

Since $\tilde{L}$ is TP we have that $\operatorname{det}((Q \tilde{L})[\gamma \mid 1,2, \ldots, k])$ is

(a) equal to 0 , if $2 \notin \gamma$.

(b) equal to $-\operatorname{det}\left((Q \tilde{L})\left[\gamma_{3}, \ldots, \gamma_{k} \mid 3, \ldots, k\right]\right) \leq 0$, if $2 \in \gamma$ and $1 \notin \gamma$.

(c) equal to $-\operatorname{det}\left((Q \tilde{L})\left[\gamma_{2}, \ldots, \gamma_{k} \mid 2, \ldots, k\right]\right) \leq 0$, if $2 \in \gamma$ and $1 \notin \gamma$.

Since $L$ is also TP, then

$$
\operatorname{det}(T[\alpha \mid 1,2, \ldots, k]) \leq 0, \quad \forall \alpha \in \mathcal{Q}_{k, n}, k=2,3, \ldots, n .
$$

Therefore, $A$ can be factorized as

$$
A=T \bar{D} U
$$

where $U$ is a unit upper triangular TP matrix, $\bar{D}=\operatorname{diag}\left(-d_{1},-d_{2}, d_{3}, \ldots, d_{n}\right)$, with $d_{i}>0$, for $i=1,2, \ldots, n$, and $T$ is the block lower triangular matrix

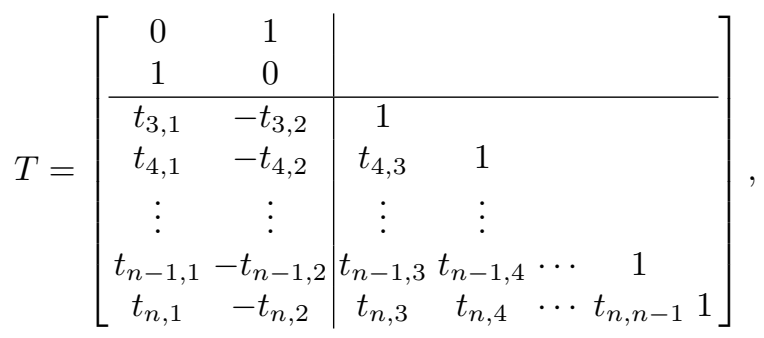

such that $T[3,4, \ldots, n]$ is a TP matrix and $\operatorname{det}(T[\alpha \mid 1,2, \ldots, k]) \leq 0$, for all $\alpha \in \mathcal{Q}_{k, n}, k=2,3, \ldots, n$. By [7, Theorem 2], then $A$ is a t.n.p. matrix with $a_{11}=0$.

Next, we extend the notation given in Definition 1 to nonsingular t.n.p. matrices with a zero $(1,1)$-entry.

Definition 2 The unique bidiagonal decomposition of an $n \times n$ nonsingular t.n.p. matrix A with $a_{11}=0$, given in Theorem 3 , is denoted by $\mathcal{B D}_{(\text {zero })}(A)$ and stored in the $n \times n$ matrix

$$
\mathcal{B D}_{(\text {zero })}(A)=\left[\begin{array}{cccccc}
d_{1} & \alpha_{1,2} & \alpha_{1,3} & \cdots & \alpha_{1, n-1} & \alpha_{1, n} \\
0 & d_{2} & \alpha_{2,3} & \cdots & \alpha_{2, n-1} & \alpha_{2, n} \\
\beta_{1,3} & \beta_{2,3} & d_{3} & \cdots & \alpha_{3, n-1} & \alpha_{3, n} \\
\vdots & \vdots & \vdots & & \vdots & \vdots \\
\beta_{1, n-1} & \beta_{2, n-1} & \beta_{3, n-1} & \cdots & d_{n-1} & \alpha_{n-1, n} \\
\beta_{1, n} & \beta_{2, n} & \beta_{3, n} & \cdots & \beta_{n-1, n} & d_{n}
\end{array}\right]
$$


where

$$
\begin{aligned}
d_{i} & >0, \quad \beta_{i, j} \geq 0, \quad \alpha_{i, j} \geq 0 \quad \text { for } i, j=1,2, \ldots, n, \\
\beta_{1, i} & >0, \quad \alpha_{1, j}>0 \quad \text { for } i=3,4, \ldots, n, j=2,3, \ldots, n, \\
\text { if } \quad \beta_{i, j} & =0, \quad\left(\alpha_{i, j}=0\right) \Longrightarrow \beta_{i, h}=0, \quad\left(\alpha_{i, h}=0\right) \text { for all } h>j .
\end{aligned}
$$

Similar to Example 1 for the case $a_{11}<0$, an $n \times n$ matrix $\mathcal{B D}_{(\text {zero })}(A)$, with the conditions given by (12), (13) and (14), is not always the bidiagonal factorization of a t.n.p. matrix $A$ with $a_{11}=0$. To know if $A$ is t.n.p. we compute $a_{n n}$ directly from the elements of $\mathcal{B D}_{(\text {zero })}(A)$. If we obtain $a_{n n} \leq 0$ then $A$ is t.n.p.

First of all, we give Algorithm 3, similar to Algorithm 1, that computes the last row of $L_{(z e r o)}$. The elements $l_{n, j}^{(z e r o)}$, for $j=n-1, n-2, \ldots, 3$, are computed as in Algorithm 1, but the elements $l_{n, 2}^{(z e r o)}$ and $l_{n, 1}^{(z e r o)}$ are obtained in the following way:

$$
\begin{aligned}
& l_{n, 2}^{(z e r o)}=\beta_{2,3}\left(h_{1}^{(3)}+h_{2}^{(3)}\right) \\
& l_{n, 1}^{(z e r o)}=-\beta_{1,3} h_{1}^{(3)}
\end{aligned}
$$

Then, we have got the following algorithm of, at most, $\mathbb{O}\left(n^{2}\right)$.

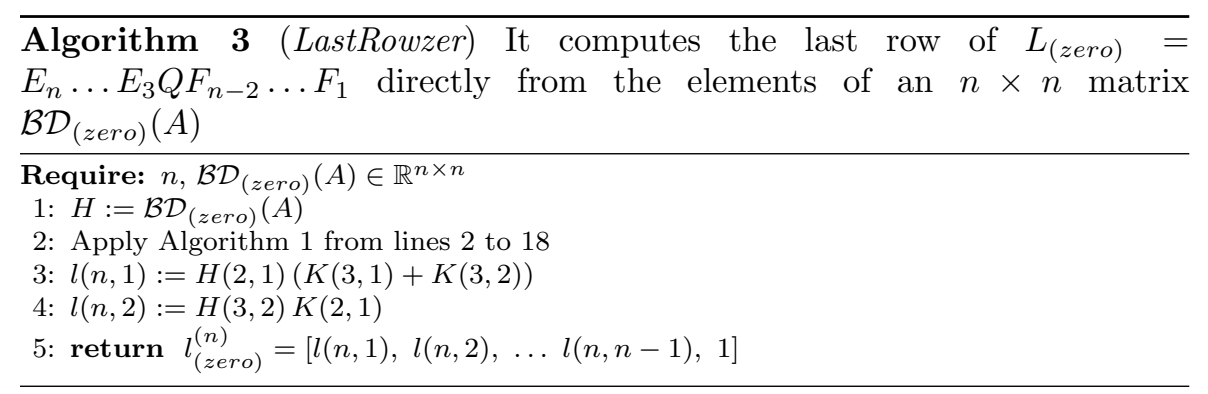

Now, we present the next algorithm, which is basically Algorithm 2 applied to $\mathcal{B D}_{(\text {zero })}(A)$ with the command LastRowzer $(H, n)$ instead of $\mathcal{B D}_{\text {t.n.p. }}(A)$ with the command $\operatorname{LastRow}(H, n)$. 


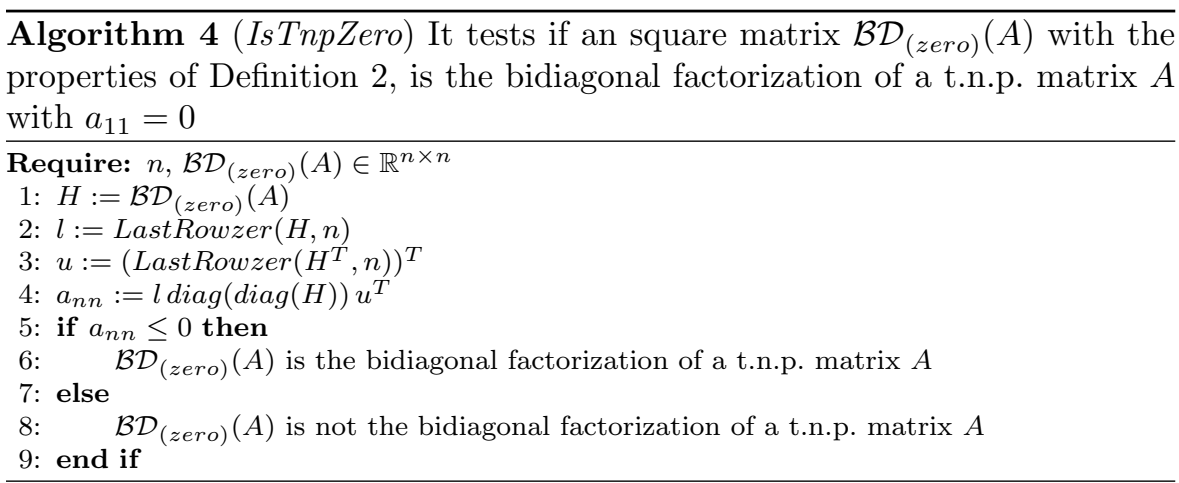

Corollary 4 The computational cost of Algorithm 4 is $3 n^{2}-8 n+5$.

In this Section we have obtained a characterization of the nonsingular t.n.p. matrices from its bidiagonal factorization, extending the known results for TP matrices. Algorithms 2 and 4 give an effective procedure to check if a matrix with the properties of Definition 1 or Definition 2 is the bidiagonal factorization of a t.n.p. matrix $A$ with $a_{11}<0$ or $a_{11}=0$, respectively.

\section{Bidiagonal factorization of the inverse of a t.n.p. matrix}

In this section, we compute the bidiagonal factorization of the inverse of a nonsingular t.n.p. matrix $A$ directly from $\mathcal{B D}_{(\text {t.n.p. })}(A)$ or $\mathcal{B D}_{(\text {zero })}(A)$.

Theorem 5 Let $A \in \mathbb{R}^{n \times n}$ be a nonsingular t.n.p. matrix, then $A^{-1}$ can be uniquely factorized as

1. If $a_{11}<0$,

$$
A^{-1}=U_{n-1} U_{n-2} \ldots U_{1} D_{(n e g)} L_{1} L_{2} \ldots L_{n-2} L_{n-1}
$$

2. If $a_{11}=0$,

$$
A^{-1}=U_{n-1} U_{n-2} \ldots U_{1} D_{(\text {zero })} L_{1} L_{2} \ldots L_{n-2} Q L_{(\text {zero })}
$$

where $D_{(\text {neg })}=\operatorname{diag}\left(-1 / d_{1}, 1 / d_{2}, \ldots, 1 / d_{n}\right), D_{(z \text { ero })}=\operatorname{diag}\left(1 / d_{1}, 1 / d_{2}, \ldots, 1 / d_{n}\right)$, with $d_{i}>0$ for $i=1,2, \ldots, n$.

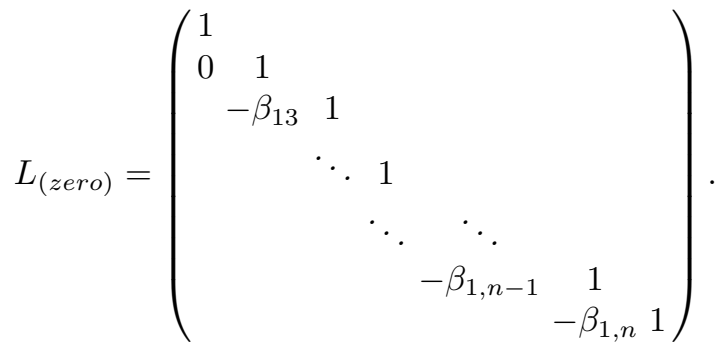


For $j=1,2, \ldots, n-1$,

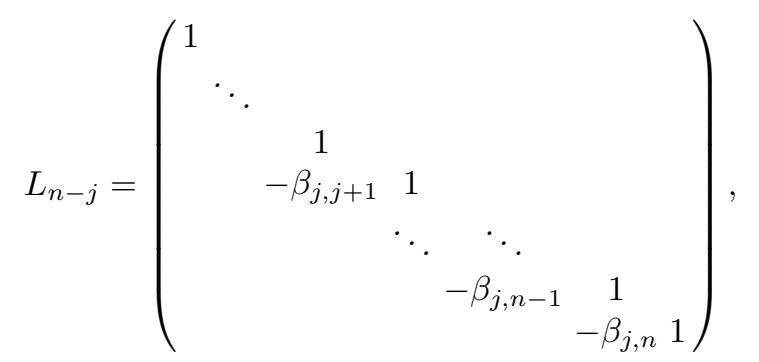

and for $k=1, \ldots, n-1$,

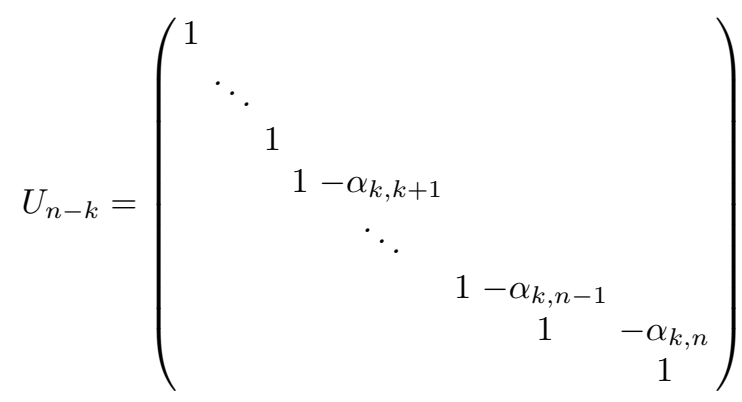

where the elements $\alpha_{j k}, \beta_{j k}$ and $d_{i}$ are the corresponding entries of $B D_{(t . n . p .)}(A)$ or $B D_{(z e r o)}(A)$.

Proof 1. If $a_{11}<0$ we know by [4] that $A$ admits the factorization $A=$ $L D U$, where $L(U)$ is a unit lower (upper) triangular TP matrix, and $D=$ $\operatorname{diag}\left(-d_{1}, d_{2}, \ldots, d_{n}\right)$, with $d_{i}>0$ for $i=1,2, \ldots, n$. Since $L$ and $U$ are TP by applying [12] we have that

$$
L=\prod_{j=1}^{n-1}\left(\prod_{i=n}^{j+1} E_{i}\left(\beta_{j i}\right)\right) \quad \text { and } \quad U=\prod_{j=n-1}^{1}\left(\prod_{i=n}^{j+1} E_{i}^{T}\left(\alpha_{j i}\right)\right)
$$

where $\beta_{1,2} \geq 0, \ldots, \beta_{1, n} \geq 0, \alpha_{1,2} \geq 0, \ldots, \alpha_{1, n} \geq 0$ and if $\beta_{s, t}=0$ then $\beta_{s, h}=0, \forall h>t$, and if $\alpha_{s, t}=0$ then $\alpha_{s, h}=0, \forall h>t$. From these expressions and reordering the corresponding products we obtain

$$
\begin{aligned}
L^{-1}= & {\left[E_{n}\left(-\beta_{n-1, n}\right)\right]\left[E_{n-1}\left(-\beta_{n-2, n-1}\right) E_{n}\left(-\beta_{n-2, n}\right)\right] \ldots } \\
& \ldots\left[E_{2}\left(-\beta_{1,2}\right) E_{3}\left(-\beta_{1,3}\right) \ldots E_{n}\left(-\beta_{1, n}\right)\right] \\
U^{-1}= & {\left[E_{n}^{T}\left(-\alpha_{1, n}\right) E_{n-1}^{T}\left(-\alpha_{1, n-1}\right) \ldots E_{2}^{T}\left(-\alpha_{1,2}\right)\right]\left[E_{n}^{T}\left(-\alpha_{2, n}\right) \cdots E_{3}^{T}\left(-\alpha_{2,3}\right)\right] \ldots } \\
& \ldots\left[E_{n}^{T}\left(-\alpha_{n-2, n}\right) E_{n-1}^{T}\left(-\alpha_{n-2, n-1}\right)\right]\left[E_{n}^{T}\left(-\alpha_{n-1, n}\right)\right]
\end{aligned}
$$

Multiplying the $\left(E_{i}\right)$ 's inside the parentheses we obtain the bidiagonal matrices

$$
\begin{aligned}
L_{n-j} & =E_{j+1}\left(-\beta_{j, j+1}\right) E_{j+2}\left(-\beta_{j, j+2}\right) \ldots E_{n}\left(-\beta_{j, n}\right) \\
U_{n-k} & =E_{n}^{T}\left(-\alpha_{k, n}\right) E_{n-1}^{T}\left(-\alpha_{k, n-1}\right) \ldots E_{k+1}^{T}\left(-\alpha_{k, k+1}\right)
\end{aligned}
$$


given by expressions (17) and (18). Therefore,

$$
A^{-1}=(L D U)^{-1}=\underbrace{U_{n-1} U_{n-2} \ldots U_{1}}_{U^{-1}} \underbrace{D_{(n e g)}}_{D^{-1}} \underbrace{L_{1} L_{2} \ldots L_{n-2} L_{n-1}}_{L^{-1}} .
$$

2. If $a_{11}=0$ by Corollary 3 we know that $A$ can be factorized as $A=L Q \tilde{L} D U$, where $L=E_{n}\left(\beta_{1, n}\right) E_{n-1}\left(\beta_{1, n-1}\right) \ldots E_{3}\left(\beta_{1,3}\right)$, with $\beta_{1,3} \geq 0, \ldots, \beta_{1, n} \geq 0$, $D=\operatorname{diag}\left(d_{1}, d_{2}, \ldots, d_{n}\right)$, with $d_{i}>0$, for $i=1,2, \ldots, n$, and $\tilde{L}(U)$ is a unit lower (upper) triangular TP matrix. Applying [12] we have

$$
\tilde{L}=\prod_{j=2}^{n-1}\left(\prod_{i=n}^{j+1} E_{i}\left(\beta_{j i}\right)\right) \quad \text { and } \quad U=\prod_{j=n-1}^{1}\left(\prod_{i=n}^{j+1} E_{i}^{T}\left(\alpha_{j i}\right)\right)
$$

such that, $\alpha_{1,2} \geq 0, \ldots, \alpha_{1, n} \geq 0$ and if $\beta_{s, t}=0$ then $\beta_{s, h}=0, \forall h>t$, and if $\alpha_{s, t}=0$ then $\alpha_{s, h}=0, \forall h>t$. Similar to the above case we obtain that

$$
A^{-1}=\underbrace{U_{n-1} U_{n-2} \ldots U_{1}}_{U^{-1}} \underbrace{D_{(z e r o)}}_{D^{-1}} \underbrace{L_{1} L_{2} \ldots L_{n-2}}_{\tilde{L}^{-1}} Q \underbrace{L_{(z e r o)}}_{L^{-1}} .
$$

With an analogous reasoning, a similar factorization is obtained for the inverse of a t.n. matrices with all parameters $\alpha_{i, j}$ and $\beta_{i, j}$ positive.

Corollary 5 Let $A=\left(a_{i j}\right) \in \mathbb{R}^{n \times n}$ be a t.n. matrix, then $A^{-1}$ can be factorized as

$$
A^{-1}=U_{n-1} U_{n-2} \ldots U_{1} D_{(n e g)} L_{1} L_{2} \ldots L_{n-2} L_{n-1}
$$

where $D_{(\text {neg })}=\operatorname{diag}\left(-1 / d_{1}, 1 / d_{2}, \ldots, 1 / d_{n}\right)$, and the elements $\alpha_{j k}, \beta_{j k}$ and $d_{i}$ are the corresponding entries of $B D_{(t . n .)}(A)$.

In the next definition we denote by $\mathcal{B D}_{(\text {t.n.p.) }}\left(A^{-1}\right)$ or $\mathcal{B D}_{(\text {zero })}\left(A^{-1}\right)$ the bidiagonal factorization of the inverse of a t.n.p. matrix $A$ given by equation (15) or (16), depending on the $(1,1)$-entry is $a_{11}<0$ or $a_{11}=0$. We also introduce $\mathcal{B} \mathcal{D}_{(t . n .)}\left(A^{-1}\right)$ for t.n. matrices.

Definition 3 1. The unique bidiagonal decomposition of the inverse of an $n \times n$ nonsingular t.n.p. matrix A, with $a_{11}<0$, given in Theorem 5 by equation (15), is denoted by $\mathcal{B D}_{(t . n . p .)}\left(A^{-1}\right)$ and stored in the $n \times n$ matrix

$$
\mathcal{B D}_{(\text {t.n.p.) }}\left(A^{-1}\right)=\left[\begin{array}{cccccc}
-1 / d_{1} & -\beta_{1,2} & -\beta_{1,3} & \cdots & -\beta_{1, n-1} & -\beta_{1, n} \\
-\alpha_{1,2} & 1 / d_{2} & -\beta_{2,3} & \cdots & -\beta_{2, n-1} & -\beta_{2, n} \\
-\alpha_{1,3} & -\alpha_{2,3} & 1 / d_{3} & \cdots & -\beta_{3, n-1} & -\beta_{3, n} \\
\vdots & \vdots & \vdots & & \vdots & \vdots \\
-\alpha_{1, n-1} & -\alpha_{2, n-1} & -\alpha_{3, n-1} & \cdots & 1 / d_{n-1} & -\beta_{n-1, n} \\
-\alpha_{1, n} & -\alpha_{2, n} & -\alpha_{3, n} & \cdots & -\alpha_{n-1, n} & 1 / d_{n}
\end{array}\right]
$$

with positive main diagonal except for the $(1,1)$-entry equal to $-1 / d_{1}<0$, and $\mathcal{B D}_{(t . n . p .)}\left(A^{-1}\right)(i, j) \leq 0$ for all $i, j=1,2, \ldots, n$, with $i \neq j$. 
If $A$ is a t.n. matrix then the bidiagonal decomposition of its inverse $\mathcal{B D}_{(t . n .)}\left(A^{-1}\right)$ has positive main diagonal except for the $(1,1)$-entry equal to $-1 / d_{1}<0$ and $\mathcal{B D}_{(t . n .)}\left(A^{-1}\right)(i, j)<0$ for all $i, j=1,2, \ldots, n$, with $i \neq j$.

2. The unique bidiagonal decomposition of the inverse of an $n \times n$ nonsingular t.n.p. matrix A, with $a_{11}=0$, given in Theorem 5 by equation (16), is denoted by $\mathcal{B D}_{(\text {zero })}\left(A^{-1}\right)$ and stored in the $n \times n$ matrix

$$
\mathcal{B D}_{(\text {zero })}\left(A^{-1}\right)=\left[\begin{array}{cccccc}
1 / d_{1} & 0 & -\beta_{1,3} & \cdots & -\beta_{1, n-1} & -\beta_{1, n} \\
-\alpha_{1,2} & 1 / d_{2} & -\beta_{2,3} & \cdots & -\beta_{2, n-1} & -\beta_{2, n} \\
-\alpha_{1,3} & -\alpha_{2,3} & 1 / d_{3} & \cdots & -\beta_{3, n-1} & -\beta_{3, n} \\
\vdots & \vdots & \vdots & & \vdots & \vdots \\
-\alpha_{1, n-1} & -\alpha_{2, n-1} & -\alpha_{3, n-1} & \cdots & 1 / d_{n-1} & -\beta_{n-1, n} \\
-\alpha_{1, n} & -\alpha_{2, n} & -\alpha_{3, n} & \cdots & -\alpha_{n-1, n} & 1 / d_{n}
\end{array}\right]
$$

with positive main diagonal and $\mathcal{B D}_{(\text {t.n.p. }}(A)(i, j) \leq 0$ for all $i, j=1,2, \ldots, n$, with $i \neq j$.

\section{Rectangular t.n.p. matrices}

Given an $n \times m$ matrix $A$ with rank $r$, it is said that $A$ satisfies the WRC condition when it can be transformed into diagonal form by Neville elimination without permutations of rows or columns. This property allows us to compute the $L D U$ factorization of $A$, applying Neville elimination without pivoting, where $L$ and $U$ also satisfy the WRC condition and its $r$ first rows or columns, respectively, are linearly independent.

It is known that the bidiagonal factorization of the rectangular TP matrix which satisfies the WRC condition can be compacted, see $[12,15,21]$. When $A$ is nonsingular t.n.p. matrix, then it satisfies the WRC condition and its bidiagonal factorization can be compacted as we have seen in Theorem 1.

In [5] the authors prove that if $A=\left(a_{i j}\right) \in \mathbb{R}^{n \times m}$ is a t.n.p. matrix with $a_{11}<0$ and $\operatorname{rank}(A)=r \leq \min \{n, m\}$ then, it admits a full rank decomposition in echelon form $A=L D U$, where $L \in \mathbb{R}^{n \times r}$ is a unit lower echelon TP matrix, $D=\operatorname{diag}\left(-d_{1}, d_{2}, \ldots, d_{r}\right)$ with $d_{i}>0$ for $i=1,2, \ldots, r$, $U \in \mathbb{R}^{r \times m}$ is a unit upper echelon TP matrix and $\operatorname{rank}(L)=\operatorname{rank}(U)=$ $r$. Unfortunately, if $A$ does not satisfy the WRC condition then $L$ and $U$ neither, and therefore it is not possible to give a bidiagonal factorization of $A$ in compact form. In the following theorem we give the condition that allows us characterize the rectangular t.n.p. matrices from its compact bidiagonal factorization.

Theorem 6 Let $A=\left(a_{i j}\right) \in \mathbb{R}^{n \times m}$ be a matrix with all entries negative except for a nonpositive $(n, m)$ entry, $\operatorname{rank}(A)=r \leq \min \{n, m\}$ and satisfying the WRC condition. A is a t.n.p. matrix if and only if $A$ admits a unique bidiagonal factorization

$$
A=F_{n-1} F_{n-2} \ldots F_{1}\left[\begin{array}{cc}
D & O_{r \times(m-r)} \\
O_{(n-r) \times r} & O_{(n-r) \times(m-r)}
\end{array}\right] G_{1} G_{2} \ldots G_{m-1}
$$


where $D=\operatorname{diag}\left(-d_{1}, d_{2}, \ldots, d_{r}\right)$, with $d_{i}>0$, and $F_{i}\left(G_{i}\right)$ are unit lower (upper) bidiagonal TP matrices, $i=1,2, \ldots, n$. Furthermore, for $i=1,2, \ldots$, $n-r$, we have

$$
F_{i}=E_{i+1}\left(\beta_{1, i+1}\right) E_{i+2}\left(\beta_{2, i+2}\right) \ldots E_{i+r}\left(\beta_{r, i+r}\right),
$$

and for $i=n-r+1, n-r+2, \ldots, n-1$,

$$
F_{i}=E_{i+1}\left(\beta_{1, i+1}\right) E_{i+2}\left(\beta_{2, i+2}\right) \ldots E_{n}\left(\beta_{n-j, n}\right) .
$$

with $\beta_{1,2}>0, \ldots, \beta_{1, n}>0$ and if $\beta_{s, t}=0$ then $\beta_{s, h}=0$, for all $h>t$. For $j=1,2, \ldots, m-r$ we have

$$
G_{j}=E_{j+n}^{T}\left(\alpha_{n, j+n}\right) E_{j+n-1}^{T}\left(\alpha_{n-1, j+n-1}\right) \ldots E_{j+1}^{T}\left(\alpha_{1, j+1}\right)
$$

and for $j=m-r+1, m-r+2, \ldots, m-1$,

$$
G_{j}=E_{m}^{T}\left(\alpha_{m-j, m}\right) E_{m-1}^{T}\left(\alpha_{m-j-1, m-1}\right) \ldots E_{j+1}^{T}\left(\alpha_{1, j+1}\right)
$$

with $\alpha_{1,2}>0, \ldots, \alpha_{1, m}>0$, and if $\alpha_{s, t}=0$ then $\alpha_{s, h}=0$, for all $h>t$.

Proof The proof is similar to the Theorem 1, taking into account that $A$ satisfies the WRC condition.

Example 4 Consider the t.n.p matrix,

$$
A=\left[\begin{array}{rrrrr}
-109 & -218 & -109 & -109 & -109 \\
-218 & -435 & -217 & -216 & -215 \\
-109 & -217 & -107 & -102 & -96 \\
-109 & -216 & -102 & -80 & -53 \\
-109 & -215 & -96 & -53 & 0
\end{array}\right] .
$$

$A$ admits the following full rank decomposition in echelon form

$$
A=L D U=\left[\begin{array}{lll}
1 & 0 & 0 \\
2 & 1 & 0 \\
1 & 1 & 1 \\
1 & 2 & 5 \\
1 & 3 & 10
\end{array}\right]\left[\begin{array}{ccc}
-109 & 0 & 0 \\
0 & 1 & 0 \\
0 & 0 & 1
\end{array}\right]\left[\begin{array}{llllc}
1 & 2 & 1 & 1 & 1 \\
0 & 1 & 1 & 2 & 3 \\
0 & 0 & 1 & 5 & 10
\end{array}\right] \text {. }
$$

Note that $A$ satisfies the WRC condition and therefore $L(U)$ also satisfies it. Since $L(U)$ is a TP matrix from its bidiagonal factorization in compact form we obtain the bidiagonal factorization in compact form of $A$, then

$$
\begin{aligned}
A & =F_{4} F_{3} F_{2} F_{1}\left[\begin{array}{c}
I_{3 \times 3} \\
O_{2 \times 3}
\end{array}\right] D\left[I_{3 \times 3} O_{3 \times 2}\right] G_{1} G_{2} G_{3} G_{4} \\
& =F_{4} F_{3} F_{2} F_{1}\left[\begin{array}{cc}
D & O_{3 \times 2} \\
O_{2 \times 3} & O_{2 \times 2}
\end{array}\right] G_{1} G_{2} G_{3} G_{4}
\end{aligned}
$$


where $F_{i}, G_{j} \in \mathbb{R}^{5 \times 5}$ for $i, j=1,2,3,4$, and they are defined as

$$
\begin{array}{ll}
F_{1}=E_{2}(2) E_{3}(1 / 2) E_{4}(2) & G_{1}=E_{4}^{T}(2) E_{3}^{T}(1 / 2) E_{2}^{T}(2) \\
F_{2}=E_{3}(1 / 2) E_{4}(2) E_{5}(1 / 2) & G_{2}=E_{5}^{T}(1 / 2) E_{4}^{T}(2) E_{3}^{T}(1 / 2) \\
F_{3}=E_{4}(1) E_{5}(1) & G_{3}=E_{5}^{T}(1) E_{4}^{T}(1) \\
F_{4}=E_{5}(1) & G_{1}=E_{5}^{T}(1) .
\end{array}
$$

In the next example we consider a t.n.p. matrix without the WRC condition and we show that its bidiagonal factorization can not be compacted.

Example 5 Consider the following t.n.p. matrix

$$
A=\left[\begin{array}{rrrr}
-7 & -7 & -7 & -7 \\
-14 & -14 & -14 & -14 \\
-7 & -6 & -6 & -5 \\
-7 & -5 & -5 & -3 \\
-7 & -4 & -4 & 0
\end{array}\right]
$$

which admits the full rank factorization in echelon form

$$
A=L D U=\left[\begin{array}{lll}
1 & 0 & 0 \\
2 & 0 & 0 \\
1 & 1 & 0 \\
1 & 2 & 0 \\
1 & 3 & 1
\end{array}\right]\left[\begin{array}{rrr}
-7 & 0 & 0 \\
0 & 1 & 0 \\
0 & 0 & 1
\end{array}\right]\left[\begin{array}{llll}
1 & 1 & 1 & 1 \\
0 & 1 & 1 & 2 \\
0 & 0 & 0 & 1
\end{array}\right]
$$

From this factorization, it is easily seen that $A$ does not satisfy the WRC condition. Note that $L$ can be factorized in the following form

$$
L=\underbrace{E_{5}(1) E_{4}(1) E_{3}(1 / 2) E_{2}(2)}_{\in \mathbb{R}^{5 \times 5}} C_{5}^{\{2\}} \underbrace{E_{4}(1) E_{3}(1)}_{\in \mathbb{R}^{4 \times 4}} C_{4}^{\{3\}}
$$

where $C_{s}^{\{k\}}$ is the $s \times(s-1)$ matrix obtained by removing the $k$-th column to the identity matrix $I_{s \times s}$, and $U$ is factorized as

$$
U=\underbrace{U_{3}^{T}(1)}_{\in \mathbb{R}^{3 \times 3}} F_{4}^{\{3\}} \underbrace{E_{2}^{T}(1) E_{3}^{T}(1) E_{4}^{T}(1)}_{\in \mathbb{R}^{4 \times 4}}
$$

where $F_{s}^{\{k\}}$ is the $(s-1) \times s$ matrix obtained by removing the $k$-th row to the identity matrix $I_{s \times s}$. Therefore, $A$ is given by the expresion

$A=E_{5}(1) E_{4}(1) E_{3}(1 / 2) E_{2}(2) C_{5}^{\{2\}} E_{4}(1) E_{3}(1) C_{4}^{\{3\}} D U_{3}^{T}(1) F_{4}^{\{3\}} E_{2}^{T}(1) E_{3}^{T}(1) E_{4}^{T}(1)$.

which can not be compacted as in Example 4.

Similar to Definition 1 for nonsingular t.n.p. matrices, we can extend the compact notation of the bidiagonal factorization of rectangular t.n.p. matrices satisfying the WRC condition. 
Definition 4 The unique bidiagonal decomposition of an $n \times m$ t.n.p. matrix $A$, with $a_{11}<0, \operatorname{rank}(A)=r$ and satisfying the WRC condition, given in Theorem 6 , is denoted by $\mathcal{B D}_{(\text {t.n.p.r. })}(A)$ and stored in the $n \times m$ matrix

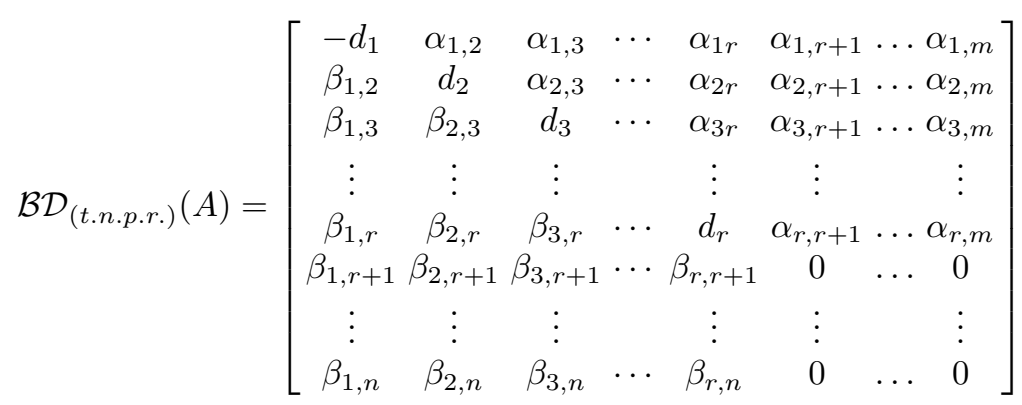

where

$$
\begin{aligned}
d_{i} & >0, \text { for } i=1,2, \ldots, r, \\
\beta_{1, i} & >0, \alpha_{1, j}>0, \text { for } i=2,3, \ldots, n, \quad j=2,3, \ldots, m,
\end{aligned}
$$

if $\beta_{s, t}=0, \quad\left(\alpha_{s, t}=0\right) \Longrightarrow \beta_{s, h}=0$, for all $h>t\left(\alpha_{s, h}=0\right.$, for all $\left.h>t\right)$.

Given an $n \times m$ matrix $\mathcal{B D}_{(\text {t.n.p.r. }}(A)$, to check when this matrix can be the bidiagonal factorization of a rectangular t.n.p. matrix $A$ with $a_{11}<0$, we need to obtain $l^{(n)}$ and $u_{(m)}$. Then, $a_{n m}$ is the product

$$
\begin{aligned}
a_{n m} & =l^{(n)}\left[\begin{array}{cc}
D & O_{r \times(m-r)} \\
O_{(n-r) \times r} & O_{(n-r) \times(m-r)}
\end{array}\right] u_{(m)} \\
& =\left[\begin{array}{llll}
l_{n, 1} & l_{n, 2} & \ldots & l_{n, r}
\end{array}\right] D\left[\begin{array}{c}
u_{1, m} \\
u_{2, m} \\
\vdots \\
u_{r m}
\end{array}\right]
\end{aligned}
$$

To compute the last row of matrix $L$ from the matrix $\mathcal{B} \mathcal{D}_{(\text {t.n.p.r. })}(A)$, we apply the Algorithm 1 if $\operatorname{rank}(A)=n$. Otherwise, we apply the following algorithm similar to Algorithm 1. 
Algorithm 5 (LastRowRectangular) It computes the last row of $L$ directly from the $n \times m$ matrix $\mathcal{B D}_{(\text {t.n.p.r. })}(A)$ with the properties of Definition 4 and with $r<n$.

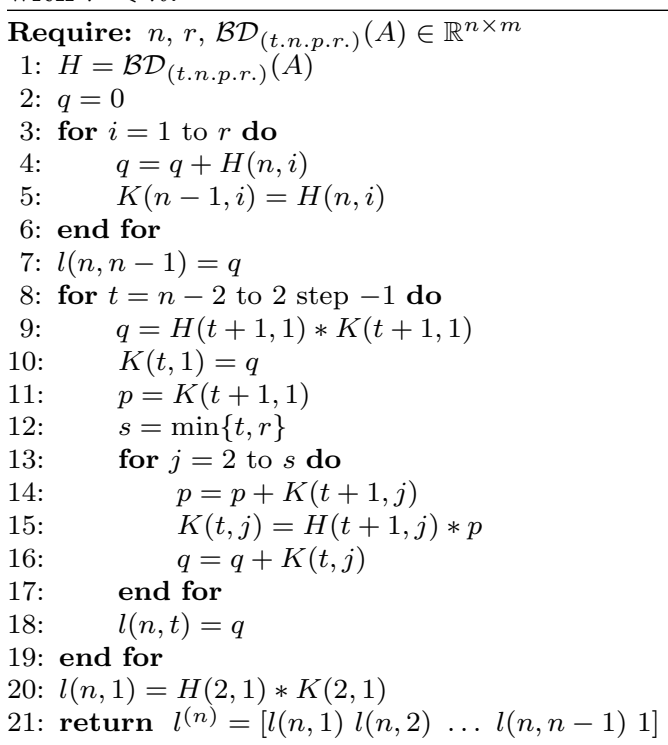

Theorem 7 The computational cost of Algorithm 5 is

- if $r<n-1$, then $O=3 n r-\frac{3}{2} r^{2}-\frac{7}{2} r-2 n+3$.

- if $r=n-1$, then $O=\frac{3}{2} n^{2}-\frac{11}{2} n+5$.

Proof The number of operations of this algorithm is:

- if $r<n-1$,

$$
\begin{aligned}
O & =(r-1)+\sum_{t=n-2}^{r}\left\{1+\sum_{j=2}^{r} 3\right\}+\sum_{t=r-1}^{2}\left\{1+\sum_{j=2}^{t} 3\right\}+1 \\
& =3 n r-\frac{3}{2} r^{2}-\frac{7}{2} r-2 n+3 .
\end{aligned}
$$

- if $r=n-1$,

$$
\begin{aligned}
O & =(n-2)+\sum_{t=n-2}^{2}\left\{1+\sum_{j=2}^{t} 3\right\}+1 \\
& =\frac{3}{2} n^{2}-\frac{11}{2} n+5 .
\end{aligned}
$$


Now the following algorithm checks when a rectangular matrix $\mathcal{B D}_{(\text {t.n.p.r. }}(A)$ with the properties of Definition 4 , can be the bidiagonal factorization of a rectangular t.n.p. matrix $A$ with $a_{11}<0$ and $r<\min \{n, m\}$. Its computational costs is $\mathbb{O}(n r)$.

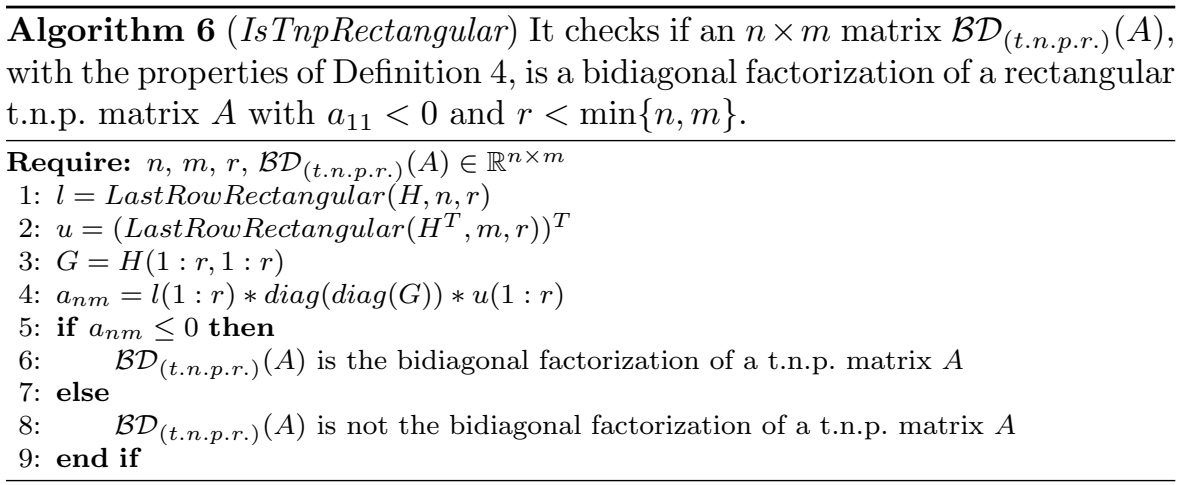

As in the square case, we obtain the following result, whose proof is straightforward

Corollary 6 The cost of Algorithm 6 is

- if $r<\min \{n-1, m-1\}$, then $O=(3 r-2)(n+m)-3 r^{2}-4 r+5$.

- if $r=n-1$, then $O=3 n m-3 n-5 m+6$.

- if $r=m-1$, then $O=3 n m-3 m-5 n+6$.

Remark 3 If $r=n$, Algorithm 6 can be applied to compute the last row of $L$, but using $l=\operatorname{LastRow}(H, n)$ instead of $l=\operatorname{LastRowRectangular}(H, n, r)$. Similarly, when $r=m$ we can used Algorithm 6 to compute the last column of $U$ replacing $u=\left(\text { LastRowRectangular }\left(H^{T}, m, r\right)\right)^{T}$ by $u=\left(\operatorname{LastRow}\left(H^{T}, m\right)\right)^{T}$.

Remark 4 If $A=\left(a_{i j}\right) \in \mathbb{R}^{n \times m}$ is a t.n. matrix, note that $a_{11}<0$ and $r=$ $\min \{n, m\}$, then we can apply Algorithm 6 taking into account the comments of Remark 3.

Remark 5 We can obtain similar results of this section when we consider a t.n.p. matrix $A=\left(a_{i j}\right) \in \mathbb{R}^{n \times m}$ with $a_{11}=0$ and $\operatorname{rank}(A)=r \leq \min \{n, m\}$.

\section{Conclusions}

We have extended the principal representation of TP (STP) matrices as products of bidiagonal matrices to nonsigular t.n.p. (t.n.) matrices and the extension to rectangular t.n.p. (t.n.) matrices with the WRC condition. From these decompositions we can compute easily the bidiagonal factorization, and therefore the inverse, of nonsingular t.n.p. (t.n.) matrices. 


\section{References}

1. T. Ando. Totally positive matrices. Linear Algebra and its Applications 90 (1987) 165219.

2. P. Alonso, J.M. Peña, M.L. Serrano. Almost strictly totally negative matrices: An algorithmic characterization. Journal of Computational and Applied Mathematics 275 (2015) 238-246.

3. R.B. Bapat, T.E.S. Raghavan. Nonnegative matrices and applications. Cambridge University Press, New York, 1997.

4. R. Cantó, P. Koev, B. Ricarte, A.M. Urbano. LDU-factorization of Nonsingular Totally Nonpositive Matrices. SIAM J. Matrix Anal. Appl. 30(2) (2008) 777-782.

5. R. Cantó, B. Ricarte, A.M. Urbano. Full rank factorization in echelon form of totally nonpositive (negative) rectangular matrices. Linear Algebra and its Applications 431 (2009) 2213-2227.

6. R. Cantó, B. Ricarte, A.M. Urbano. Characterizations of rectangular totally and strictly totally positive matrices. Linear Algebra and its Applications 432 (2010) 2623-2633.

7. R. Cantó, B. Ricarte, A.M. Urbano. Quasi- $L D U$ factorization of nonsingular totally nonpositive matrices. Linear Algebra and its Applications 439 (2013) 836-851.

8. R. Cantó, B. Ricarte, A.M. Urbano. Full rank factorization in quasi- $L D U$ form of totally nonpositive rectangular matrices. Linear Algebra and its Applications 440 (2014) 61-82 .

9. S.M. Fallat, P. Van Den Driessche. On matrices with all minors negative. Electronic Journal of Linear Algebra 7 (2000) 92-99.

10. S.M. Fallat. Bidiagonal factorizations of totally nonnegative matrices. The American Mathematical Monthly 108(8) (2001) 697-712.

11. S.M. Fallat, C.R. Johnson. Totally Nonnegative Matrices. Princeton University Press, New Jersey, 2011.

12. M. Gasca, C.A. Micchelli. Total Positivity and Applications. Math. Appl. 359, Kluwer Academic Publishers, Dordrecht, The Netherlands, 1996.

13. M. Gasca, J.M. Peña. Total positivity, $Q R$ factorization and Neville elimination. SIAM J. Matrix Anal. Appl. 4 (1993) 1132-1140.

14. M. Gasca, J.M. Peña. A test for strict sign-regularity. Linear Algebra and its Applications 197/198 (1994) 133-142.

15. M. Gasca and J. M. Peña. A matricial description of Neville elimination with applications to total positivity. Linear Algebra and its Applications, 202:33-53, 1994.

16. M. Gassó, J.R. Torregrosa. A totally positive factorization of rectangular matrices by the Neville elimination. SIAM J. Matrix Anal. Appl. 25 (2004) 86-994.

17. R. Huang, D. Chu. Total nonpositivity of nonsingular matrices. Linear Algebra and its Applications 432 (2010) 2931-2941.

18. R. Huang, D. Chu. Relative perturbation analysis for eigenvalues and singular values of totally nonpositive matrices. SIAM J. Matrix Anal. Appl. 36(2) (2015) 476-495.

19. S. Karlin. Total nonpositivity. Stanford University Press, Stanford, 1968.

20. P. Koev. Accurate eigenvalues and SVDs of totally nonnegative matrices. SIAM J. Matrix Anal. Appl. 27(1) (2005) 1-23.

21. P. Koev. Accurate computations with totally nonnegative matrices. SIAM J. Matrix Anal. Appl. 29(3) (2007) 731-751.

22. T. Parthasarathy. N-matrices. Linear Algebra and its Applications 139 (1990) 89-102.

23. J.M. Peña. Test for recognition of total positivity. SeMA Journal 62(1) (2013) 61-73.

24. A. Pinkus. Totally Positive Matrices. Cambridge University Press, 2010.

25. R. Saigal. On the class of complementary cones and Lemke's algorithm. SIAM J. Appl. Math. 23 (1972) 46-60. 